\title{
The Spectrum of Myelopathies in Children: Beyond Idiopathic Transverse Myelitis
}

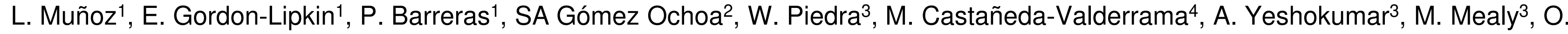
Murphy ${ }^{1}$, M. Levy ${ }^{1}$, D. Becker ${ }^{1}$, S. Newsome ${ }^{1}$, C. Pardo-Villamizar'1.

1. Johns Hopkins University, Department of Neurology, Baltimore- Maryland, USA. 2. Universidad Industrial de Santander, Department of Pediatrics, Bucaramanga, Colombia. 3. Johns Hopkins University, Department of Public Health, Baltimore- Maryland, USA. 4. Universidad Industrial de Santander, Department of Internal Medicine, Bucaramanga, Colombia.

Background and aims: Myelopathies can represent serious lifethreatening disorders, however, its heterogeneous clinical presentation and variable laboratory and radiographic features may limit significantly an early diagnosis. The objective of this study was to characterize the demographic, clinical, laboratory and imaging characteristics and evolution of the pediatric patients affected by myelopathies at Johns Hopkins Transverse Myelitis Center(1).

Methods: Analytical retrospective study. Information about the demographic characteristics, medical history, clinical presentation including the temporal profile, initial symptoms, neurologic examination and CSF profile ain 87 pediatric patients referred to a specialized myelopathy center with a diagnosis of transverse myelitis from 2010-2015 was analyzed. The final diagnosis was classified as autoimmune (AM), vascular (VM), infectious (IM), post-infectious (PIM) and metabolic myelopathy (MM).
Figure 1. Neurological symptoms temporality profile according to the etiology

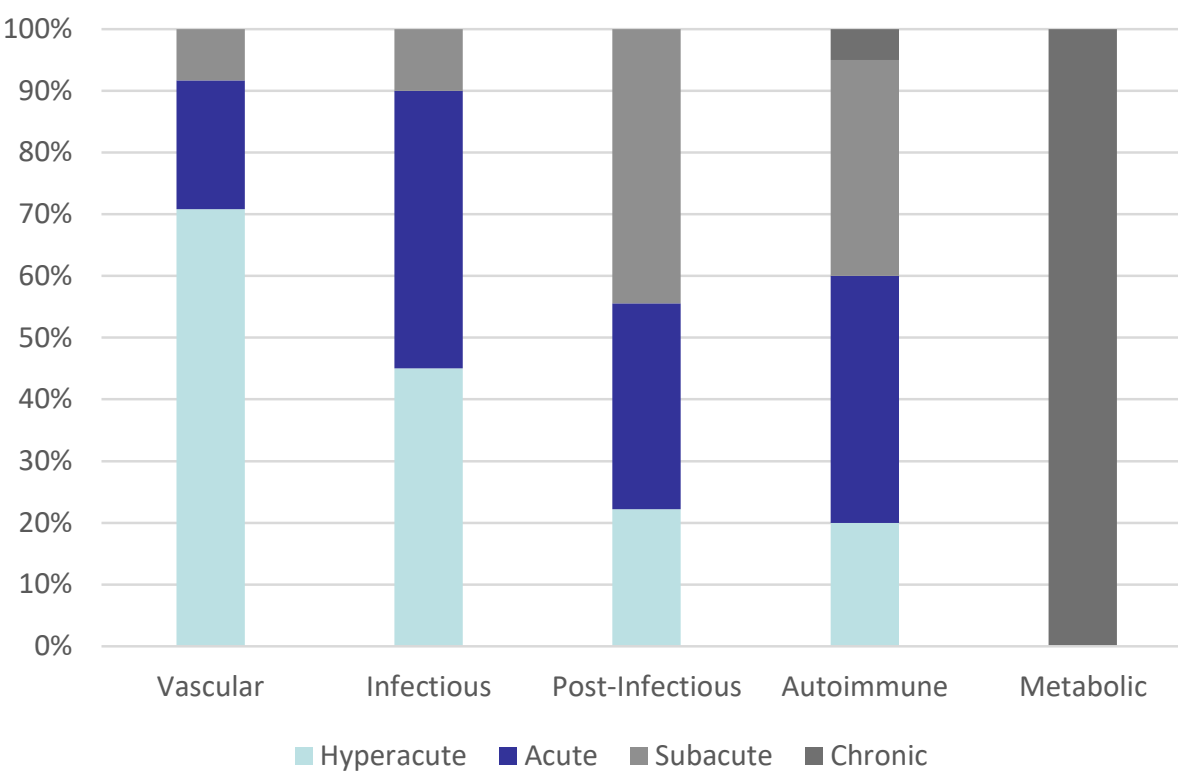

Results: Relative to VM, AM were more common in females and exhibited a more subacute profile. Patients with hypotonia, upper limbs weakness or hyporreflexia showed a lower risk of having AM with respect of the VM group. Pleocytosis increased the probability of having all the inflammatory myelopathies. The presence of an infectious disease recent history, upper respiratory symptoms at onset and pleocytosis without termoalgesia deficits had a PPV of $84,2 \%$ and a NPV of $92,6 \%$ for diagnosing an IM.

Figure 2. Clinical predictors for each diagnostic category relative to vascular myelopathies

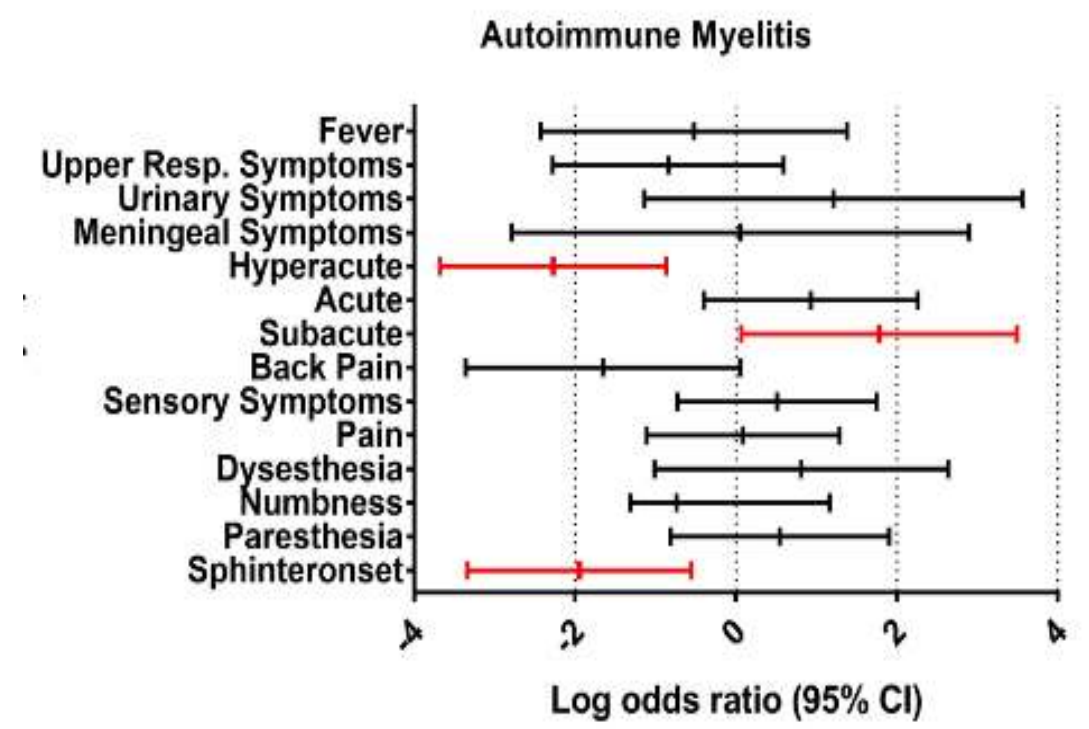

Autoimmune Myelitis

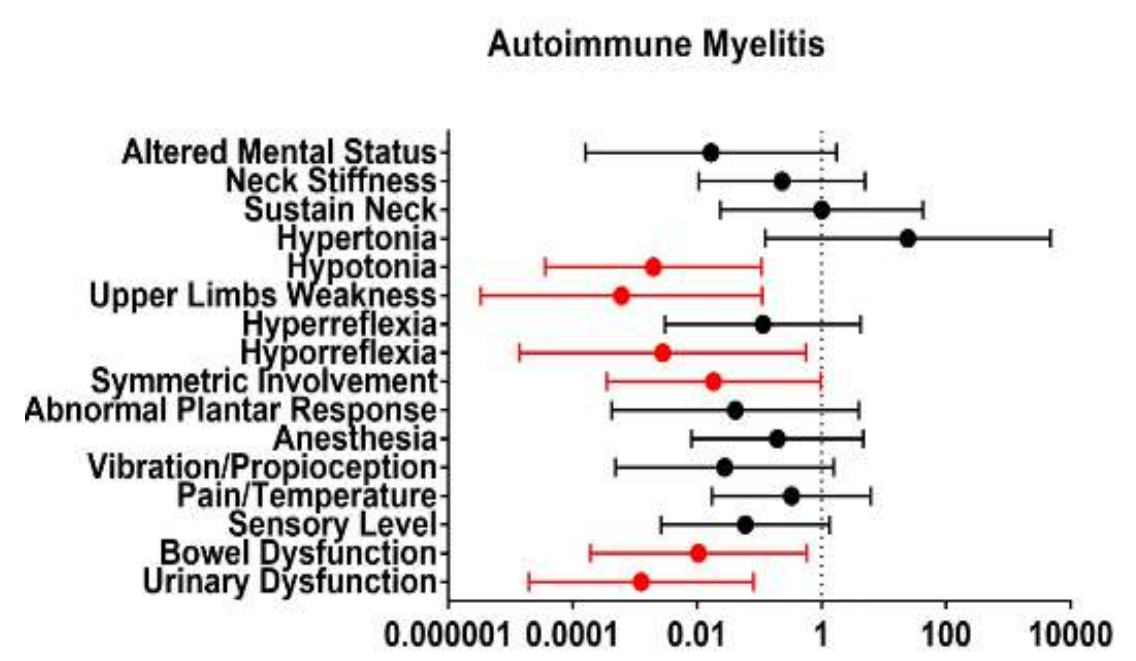

Log odds ratio $(95 \% \mathrm{Cl})$

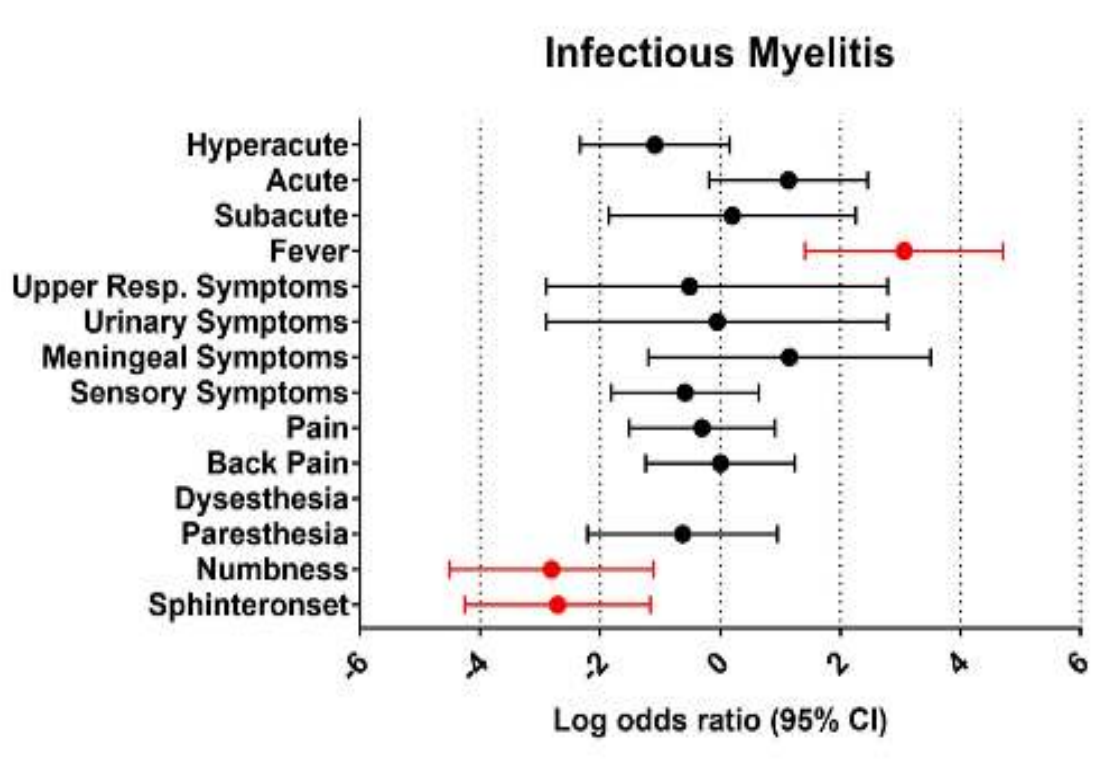

Infectious Myelitis

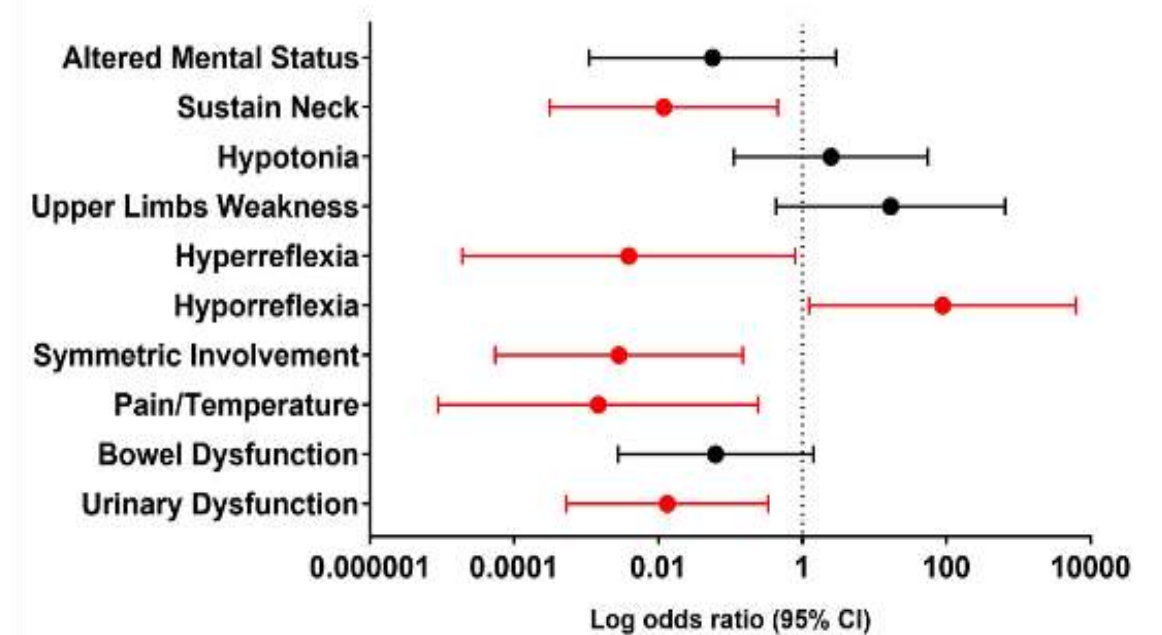

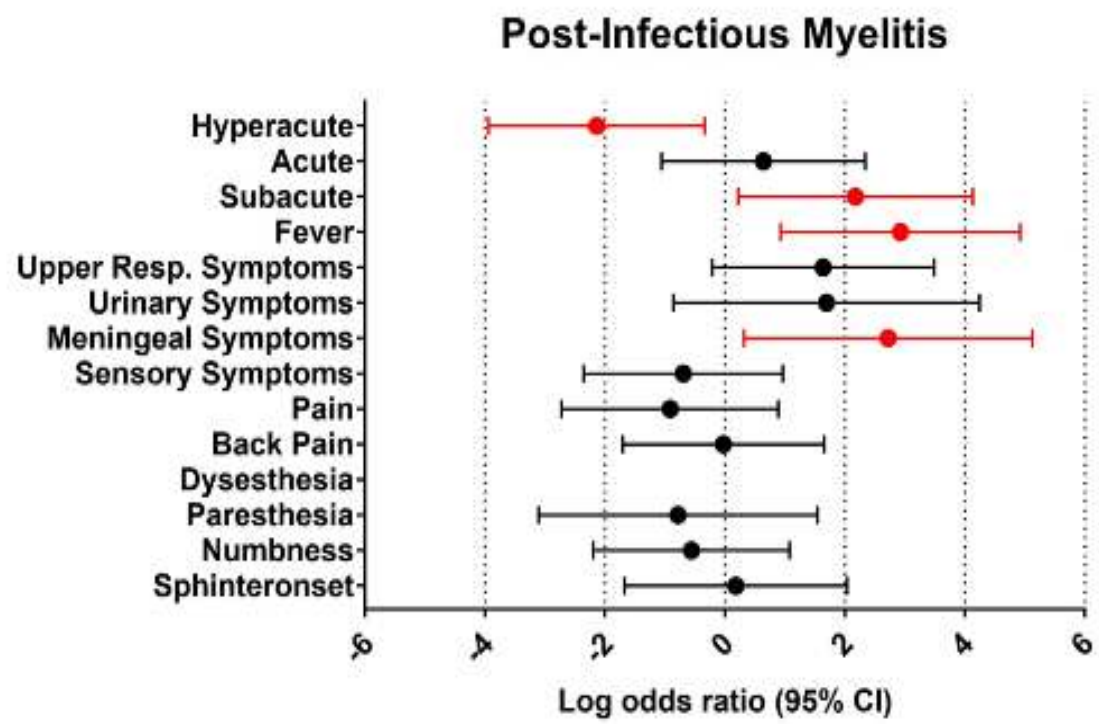

Post-Infectious Myelitis

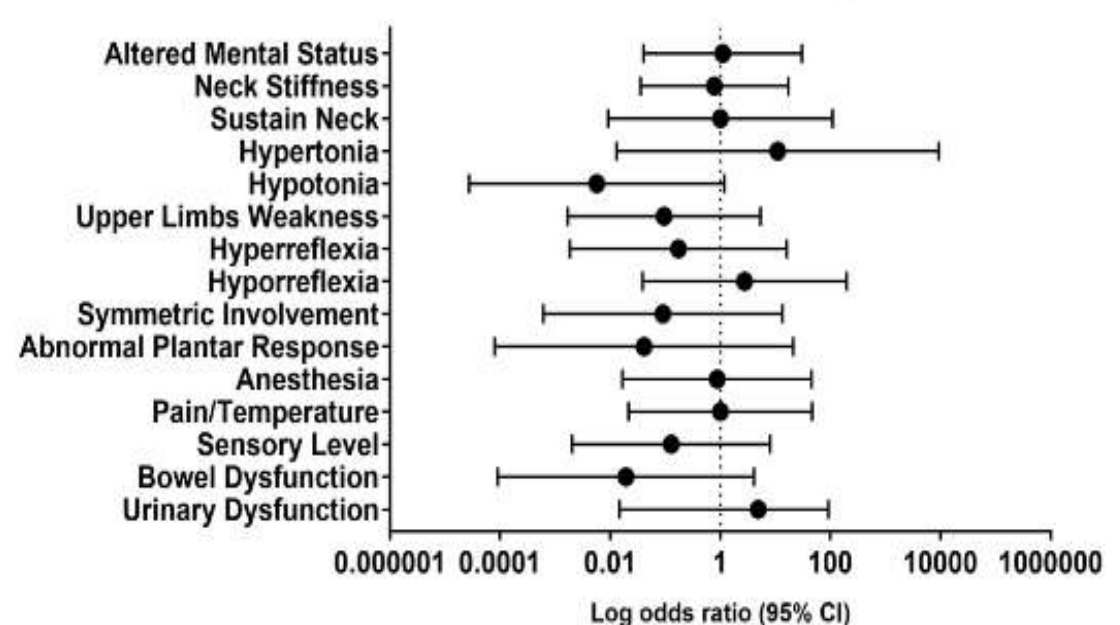

Conclusion: The spectrum of children myelopathies represent a wide variety of clinical and laboratory characteristics. Certain factors were identified to be related to specific etiologies, findings that will help identifying the type of myelopathy and favor a prompt treatment, with potential better outcomes and less sequelae.

\section{References:}

1. Barreras $P$, Fitzgerald KC, Mealy MA, Jimenez JA, Becker D, Newsome SD, Levy M, Gailloud $P 1$, Pardo CA. Clinical biomarkers differentiate myelitis from vascular and other causes of myelopathy. Neurology. 2018;90(1):e12-e21. 